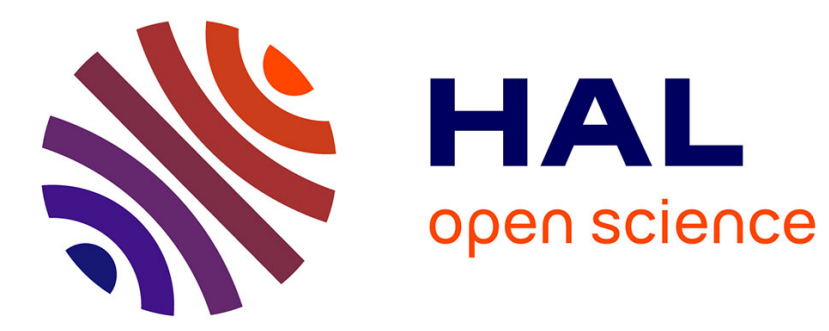

\title{
Modulateur electro-optique à onde guidée sur GaAs
}

\author{
Denis Remiens, F. Mallecot, Jean-Pierre Vilcot, Didier Decoster
}

\section{To cite this version:}

Denis Remiens, F. Mallecot, Jean-Pierre Vilcot, Didier Decoster. Modulateur electro-optique à onde guidée sur GaAs. Revue de Physique Appliquée, 1987, 22 (11), pp.1581-1584. 10.1051/rphysap:0198700220110158100 . jpa-00245710

\section{HAL Id: jpa-00245710 https://hal.science/jpa-00245710}

Submitted on 1 Jan 1987

HAL is a multi-disciplinary open access archive for the deposit and dissemination of scientific research documents, whether they are published or not. The documents may come from teaching and research institutions in France or abroad, or from public or private research centers.
L'archive ouverte pluridisciplinaire HAL, est destinée au dépôt et à la diffusion de documents scientifiques de niveau recherche, publiés ou non, émanant des établissements d'enseignement et de recherche français ou étrangers, des laboratoires publics ou privés. 
Classification

Physics Abstracts

$42.80 \mathrm{~K}-42.80 \mathrm{~L}-42.82$

\title{
Modulateur electro-optique à onde guidée sur GaAs
}

\author{
D. Remiens, F. Mallecot, J.P. Vilcot et D. Decoster
}

Centre Hyperfréquences et Semiconducteurs, U.A. CNRS 287, Université des Sciences et Techniques de Lille-Flandres-Artois, 59655 Villeneuve d'Ascq Cedex, France

(Reçu le 23 janvier 1987, révisé le 21 avril 1987, accepté le 19 mai 1987)

\begin{abstract}
Résumé. - Nous présentons un déphaseur ainsi qu'un modulateur de type interféromètre de Mach-Zehnder que nous avons réalisés sur homojonction $\mathrm{n}^{-} / \mathrm{n}^{+} \mathrm{GaAs}$ en utilisant des techniques classiques de photolithographie. La caractérisation de ces composants a montré, pour le modulateur, une tension d'extinction de $50 \mathrm{~V}$, un taux d'extinction de $10 \mathrm{~dB}$ et des pertes d'insertion de $5 \mathrm{~dB}$.
\end{abstract}

\begin{abstract}
We present a phase-shifter and a Mach-Zehnder type modulator that we have realized on $. \mathrm{n}^{-} / \mathrm{n}^{+}$GaAs homojonction using classical photolithography techniques. The characterization of the optical components has been performed and shows a $50 \mathrm{~V} \mathrm{~V}_{\pi}$ voltage, a $10 \mathrm{~dB}$ extinction ratio and $5 \mathrm{~dB}$ insertion loss for the modulator.
\end{abstract}

\section{Introduction.}

Le développement de circuits intégrés optiques associant source-modulateur-détecteur nécessite l'étude de composants optiques réalisés sur matériaux III-V. Dans la perspective de l'intégration monolithique de tels composants, guide, déphaseur, modulateur, avec des composants opto-électroniques ou hyperfréquences tels les photodétecteurs et les transistors à effet de champ sur GaAs, nous avons effectué de premières réalisations et caractérisations de composants optiques sur substrat GaAs. Dans cette communication, nous présentons une structure de guide monomode adaptée à la longueur d'onde $1,3 \mu \mathrm{m}$, un déphaseur et un modulateur électro-optique de type interféromètre de MachZehnder. Ces différents composants ont été réalisés dans la Centrale de Technologie du C.H.S et un grand nombre de caractérisations ont été menées au CNET Bagneux.

1. Définition des différentes composantes de l'interféromètre de Mach-Zehnder.

1.1 DÉfinition DU GUIDE MONOMODE. - La structure de guide monomode est obtenue sur une homojonction $\mathrm{n}^{-} / \mathrm{n}^{+} \mathrm{GaAs}$; dans ce cas, le contraste d'indice est dû à la différence de concentration de porteurs entre le substrat $\mathrm{n}^{+}\left(2 \times 10^{18} \mathrm{At} / \mathrm{cm}^{3}\right)$ et l'épitaxie de type $\mathbf{n}$ non intentionnellement dopée

REVUE DE PHYSIQUE APPLIQUÉE. - T. 22, N 11, NOVEMBRE 1987 $\left(10^{14} \mathrm{At} / \mathrm{cm}^{3}\right)$ [1]. Dans un premier temps, nous avons choisi de réaliser une structure de guide en arête (Fig.1) [2] dont la réalisation est possible avec les moyens technologiques actuellement disponibles au laboratoire (photolithographie et attaque mesa). Pour un tel guide, nous donnons le profil de mode en

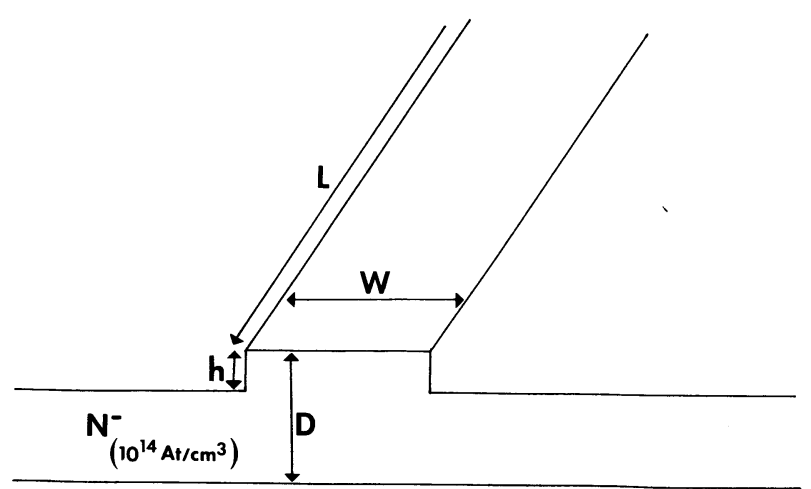

$$
\mathrm{N}^{+}\left(2 \cdot 10^{18} \mathrm{At} / \mathrm{cm}^{3}\right)
$$

Fig. 1. - Guide en arête (valeurs typiques : $W=5,5 \mu \mathrm{m}$; $h=1 \mu \mathrm{m} ; D=3 \mu \mathrm{m})$.

[Rib waveguide (typical values : $W=5.5 \mu \mathrm{m} ; h=1 \mu \mathrm{m}$; $D=3 \mu \mathrm{m})$.] 


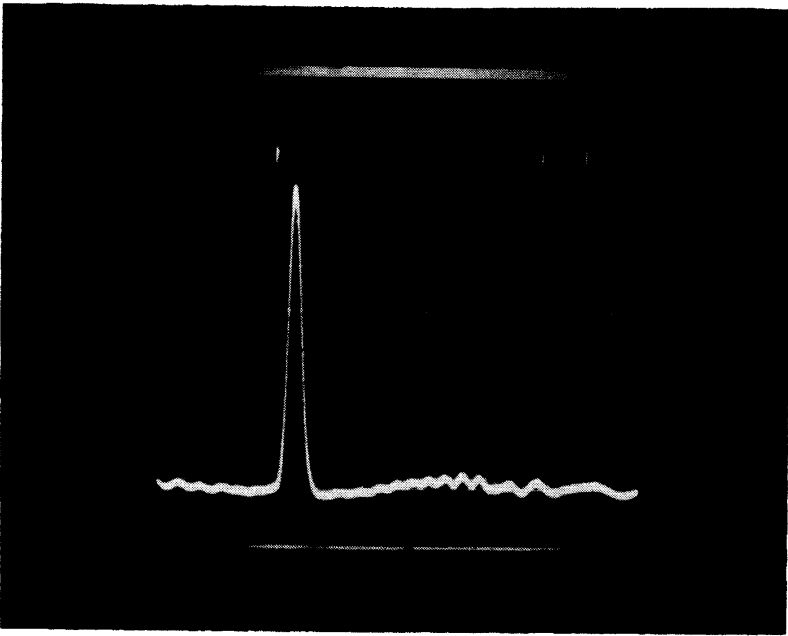

Fig. 2. - Distribution spatiale de l'intensité lumineuse en sortie de guide. Structure monomode $(D=4 \mu \mathrm{m}$; $h=1,2 \mu \mathrm{m} ; W=6,5 \mu \mathrm{m})$.

[Spatial distribution of waveguide output light. Monomode structure $(D=4 \mu \mathrm{m} ; h=1.2 \mu \mathrm{m} ; W=5.6 \mu \mathrm{m})$.]

sortie du dispositif (Fig. 2), mesuré en champ proche. Nous constatons que le guide réalisé $(D=4 \mu \mathrm{m} ; W=5,6 \mu \mathrm{m} ; h=1,2 \mu \mathrm{m})$ est bien monomode à la longueur d'onde $1,3 \mu \mathrm{m}$.

1.2 SÉPARATEur de faisceau. - Un coupleur optique $3 \mathrm{~dB}$ peut être réalisé de 2 façons différentes, soit par trois guides couplés [3], soit par couplage en Y (Fig. 3). Nous avons retenu la seconde solution, celle-ci étant la mieux adaptée à la technologie actuellement disponible au laboratoire. Nous avons étudié et réalisé plusieurs coupleurs possédant un angle d'ouverture différent. Les pertes mesurées (Fig. 4), ici définies comme le rapport de la somme des puissances de sortie du coupleur à la puissance de sortie d'un guide simple de longueur identique, deviennent très vite prohibitives lorsque l'angle d'ouverture augmente : $17 \mathrm{~dB}$ pour un angle $2 \alpha$ de
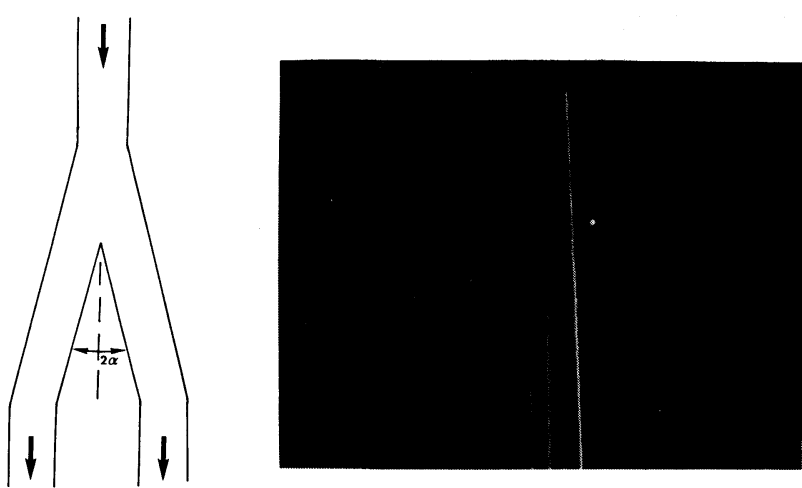

Fig. 3. - Coupleur en Y.

[Y junction.]

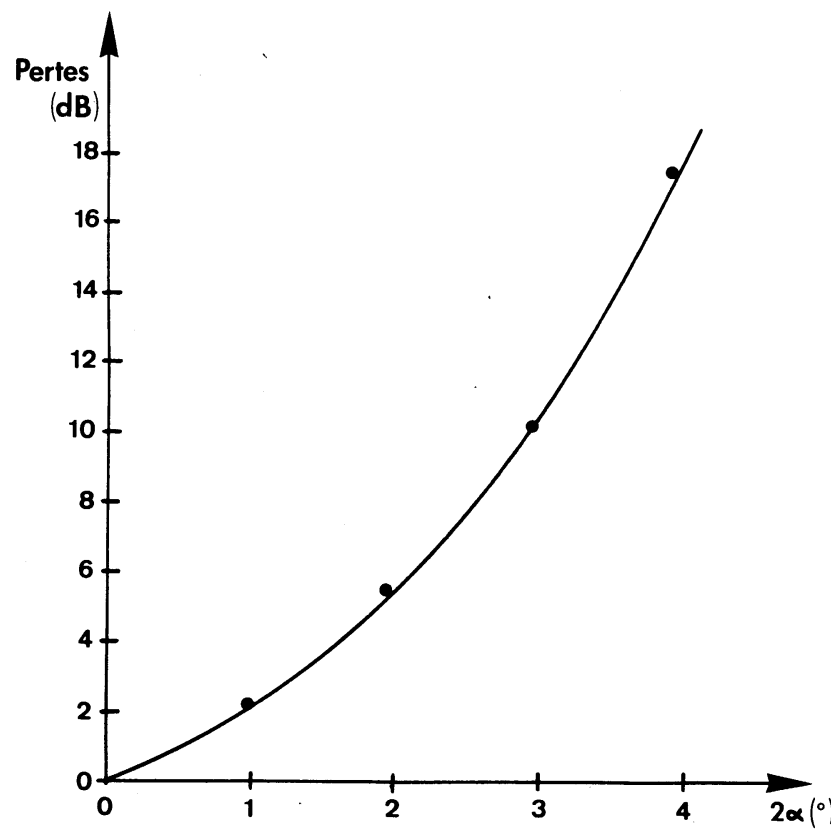

Fig. 4. - Pertes des jonctions $\mathrm{Y}$ en fonction de l'angle d'ouverture $2 \alpha(W=5,6 \mu \mathrm{m} ; h=1,1 \mu \mathrm{m}, D=4 \mu \mathrm{m})$.

[Measured loss of $\mathrm{Y}$ junctions versus aperture angle $(2 \alpha)$ $(W=5.8 \mu \mathrm{m} ; h=1.1 \mu \mathrm{m} ; D=4 \mu \mathrm{m})$.]

$4^{\circ}$. La bonne symétrie ainsi que le guidage monomode ont été vérifiés par la visualisation des intensités lumineuses de sortie (Fig. 5).

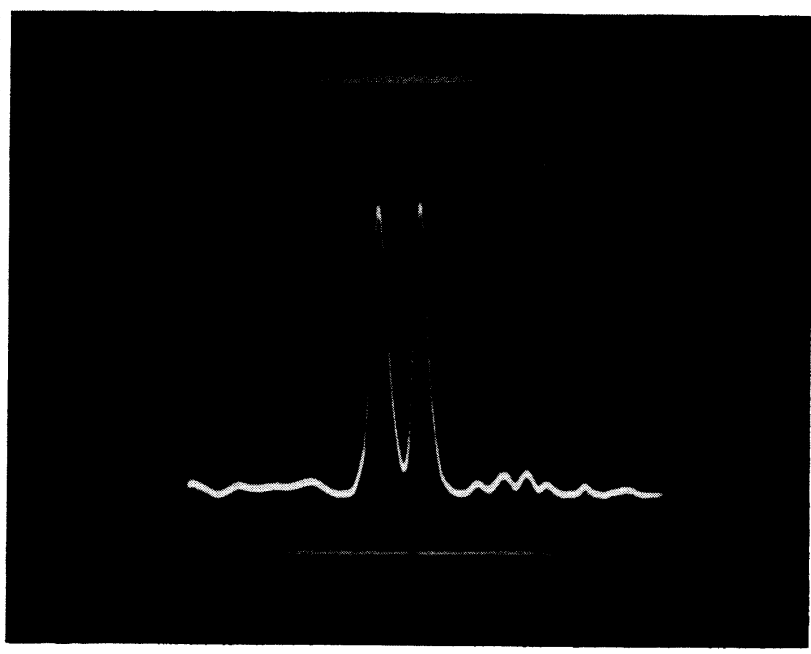

Fig. 5. - Distribution de l'intensité lumineuse en sortie de jonction Y $(W=5,3 \mu \mathrm{m} ; h=1,1 \mu \mathrm{m}, \quad D=4 \mu \mathrm{m}$; $2 \alpha=1^{\circ}$ ).

[Spatial distribution of $\mathrm{Y}$ junction output light $\left(W=5.3 \mu \mathrm{m} ; h=1.1 \mu \mathrm{m} ; D=4 \mu \mathrm{m} ; 2 \alpha=1^{\circ}\right) .1$

1.3 DéPhASEur Électro-optique. - Le déphaseur consiste en un guide en arête sur lequel a été déposée une électrode de longueur $L$. Celle-ci est réalisée par un contact Schottky : plusieurs métaux tels le platine, le titane [4] ou l'or ont été essayés. Ce 
dernier présentant de meilleures performances, notamment au niveau des pertes d'insertion, nous l'avons choisi pour la réalisation de nos dispositifs. Les pertes supplémentaires apportées par la présence du contact sont de l'ordre de $0,3 \mathrm{~dB} / \mathrm{cm}$ pour une épaisseur de métallisation de $200 \AA$; ceci confirme l'avantage de l'or vis-à-vis des autres métaux puisque des pertes de l'ordre de $5 \mathrm{~dB} / \mathrm{cm}$ ont été reportées pour le titane [4]. De plus, pour cette épaisseur de métallisation aucun phénomène d'anti-guidage n'a été observé (Fig. 6). Placé entre polariseur et analy-

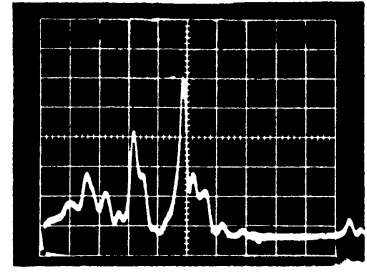

$-a-$

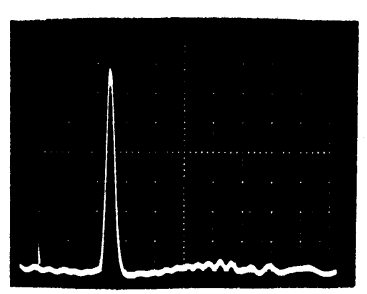

$-b-$
Fig. 6. - a) Electrode en Pt de $200 \AA$ : observation d'un phénomène d'antiguidage.

b) Electrode en $\mathrm{Au}$ de $200 \AA$ : pas d'observation de phénomène d'antiguidage.

[a) Antiguiding phenomenon for a $200 \AA$ thick Pt electrode.

b) No antiguiding phenomenon for a $200 \AA$ thick $\mathrm{Au}$ electrode.]

seur croisés, le déphaseur montre une réponse en intensité non nulle pour une tension de polarisation nulle (Fig. 7). Ceci s'explique par un effet de biréfringence naturelle de la structure, effet qui est annulé pour une tension de commande de l'ordre de $6 \mathrm{~V}$. La tension d'extinction du dispositif est de l'ordre de $40 \mathrm{~V}$, valeur qui, comparée à la tension d'extinction théorique, donne une efficacité de modulation de $87 \%$. Le rapport d'extinction, défini comme le rapport de l'intensité maximale à l'inten-

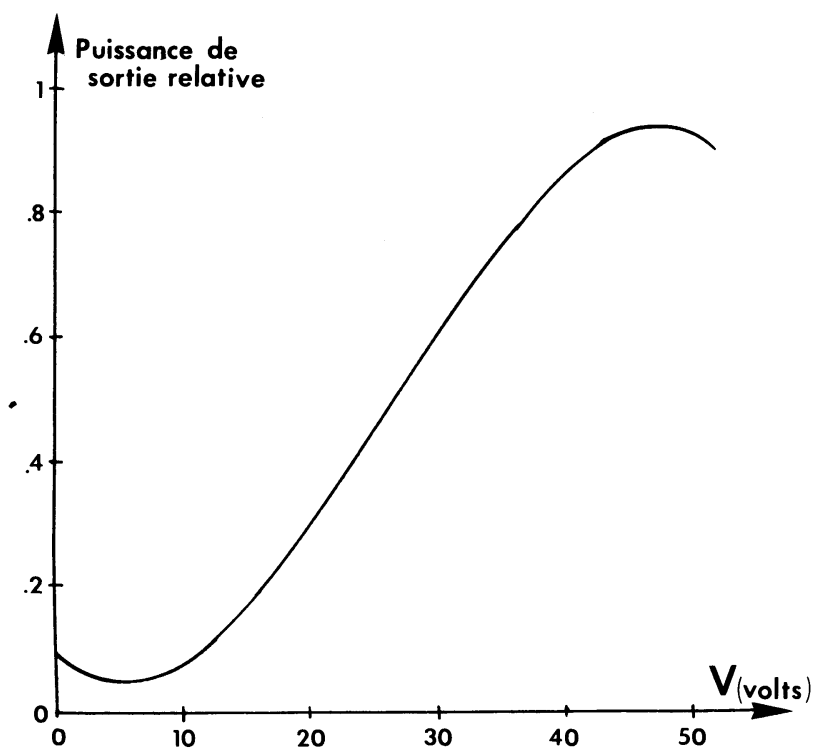

Fig. 7. - Courbe de réponse du déphaseur (polariseur et analyseur croisés).

[Dephasor response (crossed polariser and analyser).]

sité minimale détectée en sortie de guide, est de l'ordre de $14 \mathrm{~dB}$. Les pertes de propagation sont de l'ordre de $9 \mathrm{~dB} / \mathrm{cm}$, c'est-à-dire quasiment identiques à celles associées au guide non métallisé ; ce résultat confirme une fois de plus qu'aucune perte appréciable n'est engendrée par l'électrode en or.

\section{Interféromètre de Mach-Zehnder.}

Les résultats obtenus sur les précédents dispositifs ont permis de définir les paramètres géométriques d'un modulateur électro-optique de type interféromètre de Mach-Zehnder [5]. Le composant réalisé (Fig. 8) est caractérisé par un angle d'ouverture $2 \alpha$ de $1^{\circ}$, une largeur de guide $W=6 \mu \mathrm{m}$, une hauteur d'arête $h=1,2 \mu \mathrm{m}$, une épaisseur de couche guidante $D=4 \mu \mathrm{m}$ et une longueur d'électrode $L=2,8 \mathrm{~mm}$. La réponse statique (Fig. 9) est présen-
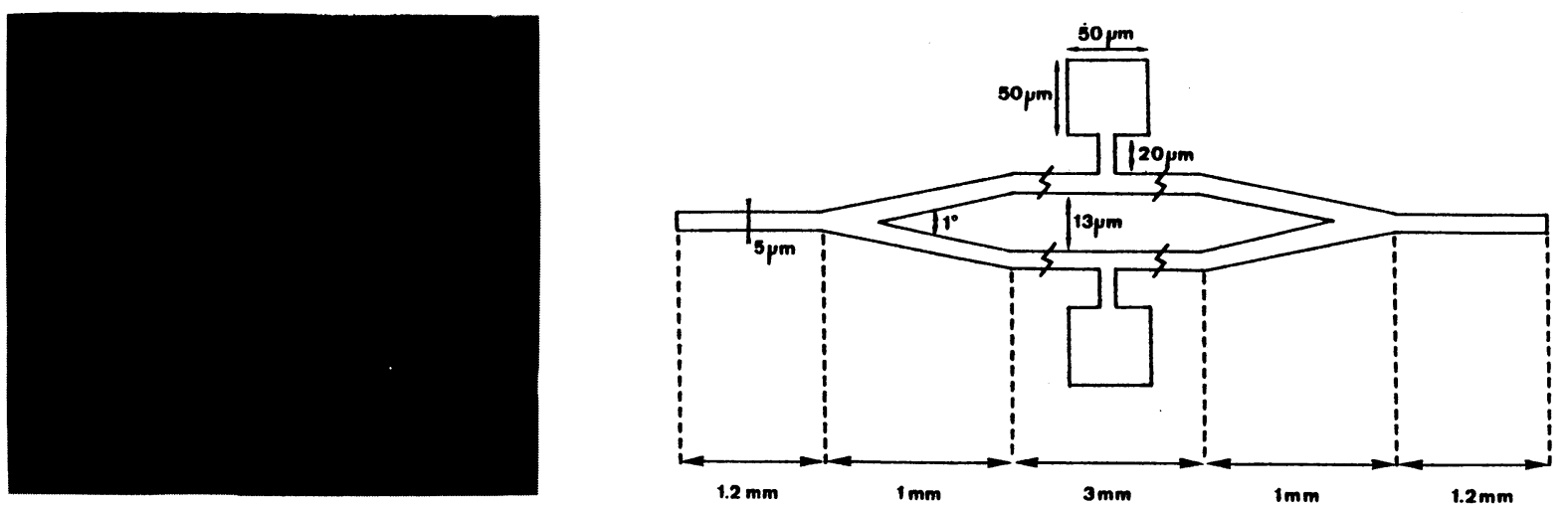

Fig. 8. - Interféromètre de Mach-Zehnder.

[Mach-Zehnder interferometer.] 


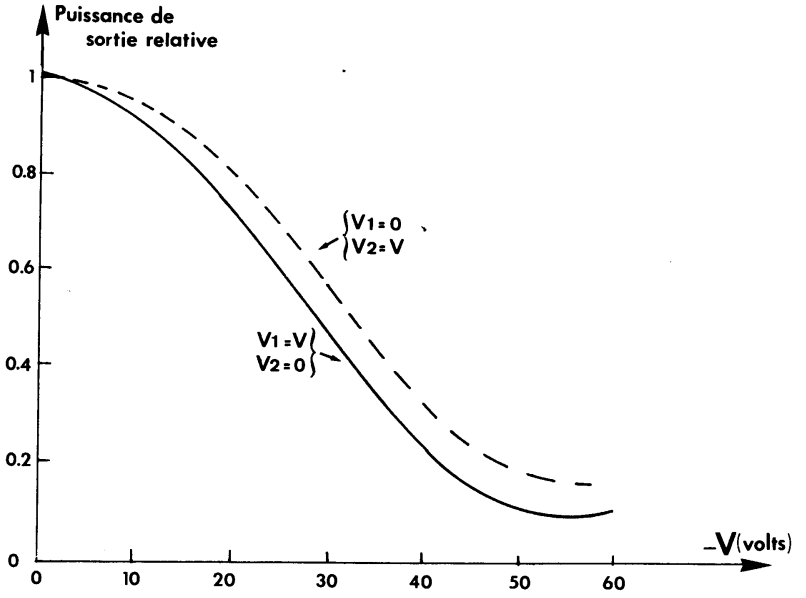

Fig. 9. - Réponse statique de l'interféromètre.

[Interferometer response.]

tée pour les deux modes de fonctionnement (tension de commande sur l'un ou l'autre bras); la différence entre les deux modes de commande peut s'expliquer par une dissymétrie entre les deux bras. La tension d'extinction est de l'ordre de $50 \mathrm{~V}$ ce qui correspond à une efficacité de modulation de $64 \%$. Le rapport d'extinction est voisin de $10 \mathrm{~dB}$. Les pertes en transmission ont été mesurées de l'ordre de $5 \mathrm{~dB}$; cette valeur provient essentiellement des jonctions $\mathrm{Y}$ et de leur écartement résiduel élevé, principalement dû aux moyens classiques de photolithographie employés. En considérant l'interféromètre comme un dispositif à structure d'électrodes localisées, la bande passante électrique a pu être indirectement évaluée par la mesure de la capacité présentée par le modulateur. Celle-ci a été mesurée, pour une tension de polarisation de $5 \mathrm{~V}$, de l'ordre de $1 \mathrm{pF}$, ce qui équivaut à une bande passante proche de $5 \mathrm{GHz}$ pour un système chargé sur $50 \Omega$ [6]. Cette façon de procéder suppose possible la représentation de l'ensemble électrode-guide sous la forme d'un circuit à constantes localisées. En toute rigueur, pour les fréquences et les longueurs d'électrodes mises en jeu, il faudrait également tenir compte des phénomè- nes de propagation hyperfréquence dans le dispositif $[7,8]$.

\section{Conclusion.}

Nous avons, en particulier, montré que, partant d'un guide en arête sur homojonction $\mathrm{n}^{-} / \mathrm{n}^{+}$, on peut obtenir, à l'aide d'une technique de photolithographie classique, des déphaseurs et modulateurs optiques. Les performances de ces dispositifs peuvent être améliorées de diverses manières. Tout d'abord, sans modifier la structure de base, l'utilisation d'un masqueur électronique devrait permettre de réduire les pertes associées aux écartements résiduels des jonctions Y. Ensuite, une réduction des pertes de propagation dans les guides peut également être obtenue en les réalisant sur des hétérostructures de type $\mathrm{GaAlAs} / \mathrm{GaAs}[9,10]$, ce qui a pour effet principal de diminuer les pertes par porteurs libres du substrat. Au delà des problèmes posés par les performances des dispositifs, il convient également d'envisager les possibilités d'intégration de ces composants optiques avec des composants optoélectroniques ou hyperfréquences. Dans ce cas, l'utilisation d'un substrat semi-isolant s'avère souhaitable et la croissance de couches de confinement de type GaAlAs serait nécessaire. En outre, on peut se demander si la réalisation de modulateurs à base de superréseaux [11] ne permettrait pas de réduire les dimensions de ces dispositifs afin de les rendre compatibles avec celles des transistors hyperfréquences. Enfin, des études développées au C.H.S. montrent la faisabilité de l'intégration monolithique de photodétecteurs sur guides optiques; les premiers résultats obtenus [12] sont prometteurs.

\section{Remerciements.}

Les auteurs tiennent à remercier messieurs A. Carenco du CNET Bagneux et M. Papuchon de Thomson L.C.R. pour les conseils et le soutien expérimental qu'ils nous ont apportés ainsi que les membres de la Centrale de Technologie du C.H.S. pour la réalisation des composants.

\section{Bibliographie}

[1] Marple, D.T.F., J. Appl. Phys. 35 (1964) 1241.

[2] Somekh, S., Garmire, E., Yariv, A., Garvin, H. et Hunsperger, R., Appl. Opt. 23 (1974) 327.

[3] Donnelly, J.P., De Meo, H.L., Ferrante, G.A., NiCHOLS, K.B., I.E.E.E. LT-1 (1983) 417.

[4] Buchmann, P., Kaufmann, H. et Melchior, H., ECOC 83, 9th European Conf. on Opt. Comm. (1983) p.539.

[5] Buchmann, P., Kaufmann, H., Melchior, H. et Gurkos, G., Appl. Phys. Lett. 46 (1985) 462.

[6] Chen, F.S., Proc. I.E.E.E. 58 (1970).

[7] TedjINI, S. et RAULY, D., 8èmes Journées Nationales d'Optique Guidée (1987).
[8] Remiens, D., Pribetich, P., Kennis, P. et SeguiNOT, C., Appl. Opt. 26 (1987) 118.

[9] Carenco, A., Sansonetti, P., Menigaux, L., Brandon, J., Rondot, M., Proc. of integrated and guided wave optics Conf. (1984).

[10] Rodgers, P.M., Proc. of the 3rd E.C.I.O. (1985) p. 117.

[11] Glick, M. et Reinhart, F.K., Proc. of the 3rd E.C.I.O. (1985) p. 99.

[12] Mallecot, F., Remiens, D., Vilcot, J.P. et Decoster, D., 8èmes Journées Nationales d'Optique Guidée (1987). 